\title{
Adaptive Channel Assignment for Wireless Mesh Networks Using Game Theory
}

\author{
Maryam Amiri Nezhad and Llorenç Cerdà-Alabern \\ Department of Computer Architecture \\ Universitat Politecnica de Catalunya, Barcelona, Spain \\ E-mail:maryam@ac.upc.edu,llorenc@ac.upc.edu
}

\begin{abstract}
Channel assignment was extensively researched for multi-radio wireless mesh networks, but still challenging when comes to implementation. In this paper we propose a semi-dynamic and distributed channel assignment called SICA based on the game theory formulation. To the best of our knowledge this is the first game formulation of channel assignment which takes the co-channel interference into account. SICA is an interference aware, distributed channel assignment which preserving the network connectivity without relaying on any common channel nor central node for coordination between mesh routers. SICA applies an on-line learner algorithm which assumes that nodes doesn't have perfect information. We have implemented SICA and compared against another interference-aware channel assignment proposed in the literature called Urban-X. Simulation results show that SICA outperforms Urban-X, even using less radio interfaces per node.
\end{abstract}

Keywords-Channel Assignment; Multi-Radio; MultiChannel; Wireless Mesh Network; Game Theory; Online Learning;

\section{INTRODUCTION}

Multiple-antenna technologies are well known to offer significant improvement in capacity through the use of multiple frequencies offered in IEEE 802.11 standards. The network capacity can be further enhanced if the network employs an intelligent channel assignment which seeks a proper mapping between the available channels and the radios at every node.

Many channel assignment approaches fell into static category, where mesh nodes tune an antenna to a specific channel permanently (see e.g., [1], [2] and references therein).Due to the variable nature of the wireless medium, the channel assignment mechanism must be flexible enough to adopt to the erratic traffic or interference pattern. Static CAs are unable to cope with the external interference but they can easily be extended to semidynamic by refreshing the channel assignment at regular time intervals in response to changes in traffic pattern or co-channel interference(see e.g. [3]-[5] and references therein).

In this work we propose SICA, a semi-dynamic interference aware channel assignment algorithm for IEEE 802.11 based WMN. We estimate the amount of interference over channels, induced by any wireless enabled devices, based on IEEE $802.11 \mathrm{k}$ standard. We then use the game theory to formulate the problem. Unlike previous game formulation in the literature we assume a more realistic scenario where nodes do not have perfect information about others strategies and channels suffer external interference from neighboring networks. Then we apply the online learning method to design a distributed algorithm which assigns the best channel to each radio. The nodes continuously refine their decision accounting the changes in the wireless environment. The main contributions that makes our work apart form others are as follows:

- A novel game theory formulation of channel assignment, considering external and internal interference.

- A decision making strategy assuming imperfect information at each router but adopts fast to the changes in the wireless environment.

- A fully distributed CA algorithm which preserves the network connectivity and supports any routing protocol.

- A self-contained protocol which apply channel load estimation, interface switching, control message exchange and data delivery mechanisms in addition to channel assignment.

We evaluate SICA through simulations using ns-3, and compare it with another distributed and interference aware channel assignment mechanism, that has been proposed in the literature. Results demonstrate the effectiveness of SICA in exploiting channel diversity, hence reducing the interference over wireless links, even with a small number of radios per node.

\section{RELATED WORK}

The channel assignment problem is well studied in recent years [6], [7] and many semi-dynamic solutions have been addressed in previous proposals [3]-[5], but few proposals consider the effect of the external interference.

The first interference aware but centralized CA is proposed in [3] where each node estimates the co-channel interference and coordinates with a central node through a common channel. The coordinator then assigns channels to links. The main drawback of the proposal is the reallocation of the channels changes the network topology and invoked the routing protocol to reroute the traffic. Urban-X is another adaptive and semi-dynamic channel assignment proposed in [4]. Urban-X is proposed for a network where each node must have at least three radios. One radio of all nodes is tuned to a common channel and is used for control traffic. The channel assignment consider the external interference in addition to the number of flows at each node, to make decision. In this paper we shall compare SICA against Urban-X.

Channel assignment algorithms using game theory models have been studied recently in some works [5], [8], 
[9]. None of the proposed algorithms considers the effect of co-channel interference. All approaches consider that nodes or players have information about all strategies and payoffs. However, in a scenario having external interference it is difficult to have the perfect knowledge of the channel occupancy before making decisions. Felegyhazi et al. [5], formulate channel assignment as a game where traffic flows compete for shared channels in a conflict situation. Although the algorithm converges to a stable Nash Equilibrium but their work is limited to single collision domain. Further extensions of this work for multi hop networks in one collision domain are presented in [8], [10]. Kim et al. [10] did not put any constraints for the number of radios per node. The proposed game should played sequentially and channels should be reallocated for any changes in the traffic profile. The approaches proposed in [8] formulates the channel assignment as a cooperative game but channel reallocation is necessary for changes in traffic sessions. Shah et al. [9] formulate the game for multiple collision domains, but they use a static game which is limited to find an equilibrium for competing flows. Unlike all previous game models for channel assignment, SICA consider co-channel interference while assuming that nodes have imperfect information and the solution is independent from the traffic profile.

\section{SICA ARCHITECTURE}

We introduce a multi-radio multi-channel architecture which reduces the impact of the wireless interference, and improves the performance of the network by driving the benefits of non-overlapping channels. Channel assignment is viewed a lower layer mechanism which doesn't consider the traffic load. Our goal is to reduce the effect of the interference inside the mesh network and with any other co-located wireless networks. The distributed multichannel architecture considers the cannel selection mechanism, describes the switching process of the antennas and controls data buffering and transmitting. Nodes use a distributed algorithm to occupy the best channel based on the information gathered during the channel sensing periods.

We shall describe SICA with nodes equipped with 2 radio interfaces, each provided with a set $C$ (with cardinality $|C|>1$ ) of non overlapping channels. However, SICA could be easily extended to a network where nodes are equipped with a number of radios larger than 2 . The radios will be referred to as the receiving radio and the transmitting radio, and denoted by $R$ and $T$, respectively. The distributed channel assignment selects and assigns the best channel to the $R$ radio of each node. Then, nodes switch the $T$ radio accordingly. For example, if a generic node $A$ tunes its $R$ radio over channel $c \in C$, each neighboring node, which aims to send traffic to $A$, will switch its $T$ radio to channel $c$ before start transmission. The $T$ radio remains on channel $c$ until all the packets addressed to node $A$ have been sent, or until a maximum period of time $\left(T_{\max }\right)$.

In the following sections we explain the details of
SICA. We explain the channel sensing mechanism, CA algorithm and its implementations in sections IV, $\mathrm{V}$ and VI, respectively. The synchronization and switching of $R$ and $T$ radios are explained in sectionsVII.

\section{EXTERNAL INTERFERENCE ESTIMATION}

To estimate the amount of external interference, mesh nodes use the clear channel assessment (CCA) mechanism for spectrum sensing [11]. CCA is based on energy detection during a specific period of time. At a given time all nodes on the same channel stop transmission and start sensing the channel, the synchronization is achieved through sending messages (see section VII). Since all nodes working on the same channel must remain silent during listening to the carrier, a big sensing period will degrade the network throughput, on the other hand, a long enough sensing period is necessary to have a precise estimation [4]

During the sensing period $\left(T_{S S}\right)$ every node monitors the channel by taking samples at the sense rate $\left(T_{\text {SRate }}\right)$. The channel status would be monitored as either idle or busy. Define $T_{i, b u s y}(c)$, the time that channel is sensed as busy during the sensing period. On the contrary $T_{i, i d l e}(c)$ shows the amount of time that the channels is sensed as idle. IEEE $802.11 \mathrm{k}$ standard for radio resources measurementproposes a simple formulation to compute the channel load as the percentage of time that the node sensed the medium as busy. At the end of sensing period node $i$ estimates the normalized bandwidth (or duty cycle) consumed by external networks over a channel $c$, as:

$$
B_{i, n e i g}(c)=\frac{T_{i, \text { busy }}(c)}{T_{i, \text { busy }}(c)+T_{i, \text { idle }}(c)}
$$

The mesh nodes then uses the channel load to make decision in channel assignment algorithm (see Section V).

\section{Channel Assignment Algorithm}

We have used a game theory model for the distributed channel assignment of SICA, which is adaptive to the external interference. In our model each node is a rational player which tries to occupy the best channel for its $R$ radio. The best channel is a channel which suffers less external interference and it is not shared by many neighboring nodes of the same network. From this point forward we use the terms node and player interchangeably.

Let $N$ be the number of nodes of the network, and $f_{i, c}$ the number of $R$ radios of player $i$ using channel $c\left(f_{i, c} \in\right.$ $\{0,1\})$. Define the strategy of player $i, s_{i}$, as its channel allocation vector, given by $s_{i}=\left(f_{i, 1}, f_{i, 2}, \ldots, f_{i,|C|}\right), i=$ $1, \cdots N$.

A player strategy describes whether it has a radio over a specific channel or not. Note that the total number of $R$ radios employed by player $i$ is given by $f_{i}=\sum_{k=1}^{C} f_{k, c}$. Since only one $R$ radio is used, $f_{i}=1$. We define the strategy matrix (strategy profile), $S$, as the strategy vector of all players at a given time: $S=\left[s_{1} s_{2} \ldots s_{N}\right]$. By $S_{-i}$ we shall refer to the strategy matrix consisting of all nodes' strategies except player $i$. Note that the node may not know $S_{-i}$ completely. 
We formulate a game theory model where each player $i$ chooses a channel $c$ trying to minimize a loss function. Each mesh router derives two separate costs fro selecting a channel. The first cost is according to the channel load estimated in Section IV(equation (1)). The second cost is according to internal interference induced from neighboring nodes. To estimate the internal interference over a channel, mesh routers compute how congested is the channel in the neighborhood. Let $N_{i}$ is the number of nodes in the interference range of node $i$ (two-hops neighbors based on interference protocol model [12]). We shall represent by $R_{i}(c)$ the number of nodes in the set $N_{i}$ that have tuned their $R$ radio to channel $c$ at a given time:

$$
R_{i}(c)=\sum_{k \in N_{i}} f_{k, c}
$$

We define the density of interfering nodes over channel $c$ by $\frac{R_{i}(c)}{N_{i}}$. The mesh router then merges the costs by taking the average of the individual cost as bandwidth loss function:

$$
M_{i, B}\left(c, S_{-i}\right)=\frac{1}{2}\left(B_{i, n e i g}(c)+\frac{R_{i}(c)}{N_{i}}\right)
$$

However, the cost of one node's decision depends not only on the available bandwidth of the selected channel, but also the switching delay penalty. According to [4], [13] current 802.11 commodities suffer a considerable switching delay $\left(D_{s}\right)$ varying from $80 \mu s$ to $22 \mathrm{~ms}$. A big switching delay of a radio affects the performance of the protocol if the radio switches frequently. We consider the magnitude of the switching delay related to the Hello interval, $T_{H}$ (explained in section ??). If the hello interval is big enough the effect of the switching delay is negligible. On the other hand a considerable switching delay should give a higher cost, making nodes to switch between channels less frequently.

Let $c_{i}$ the channel being used by node $i$, we assume that a switching delay loss function, for any channel, is given by:

$$
M_{i, D}\left(c, S_{-i}\right)= \begin{cases}\frac{D_{s}}{T_{H}}, & c \neq c_{i} \\ 0, & \text { otherwise }\end{cases}
$$

Finally, we combine bandwidth and switching delay costs in the loss function given by:

$$
M_{i}\left(c, S_{-i}\right)=\gamma M_{i, B}\left(c, S_{-i}\right)+(1-\gamma) M_{i, D}\left(c, S_{-i}\right)
$$

Note that, $\gamma$ is a tuning parameter $(\gamma \in[0,1])$, and the loss function codomain is $[0,1]$. It is not feasible nor necessary for a player to compute $M_{i}\left(c, S_{-i}\right)$ for all possible values of $S_{-i}$. Each player computes the loss value for one strategy profile at a time, in Section V-A we explain how this method solves the game effectively.

To sum up, we defined a game with the following properties:

- Nodes are rational players and try to occupy the most vacant frequency channels.

- Nodes do not have knowledge about their neighbors criteria of making decision, beforehand.
- Each channel decision imposes a cost (in the range of 0 to 1) to a node, as a function of switching delay and available bandwidth over the selected channel.

- The game is played in several rounds and the external parameters introduced by the environment may differ in each round, i.e. the environment is unpredictable.

\section{A. Solving the game}

Due to the changes in the co-channel interference, the game outlined in the previous section has no deterministic loss matrix, therefore using common approaches to solve the game is impossible. Our solution is based on the online learning approach proposed by Freund and Schapire in [14], [15].

Let $M_{i}\left(c, S_{-i}\right)$ be the loss matrix of node $i$, i.e. the rows of $M_{i}$ are the strategies of node $i$ (the channels $c \in C$ it can choose), and the columns are all possible strategies of the other players, $S_{-i}$. Each node assigns non-negative weights $\left(w_{i}(c)\right)$ to the rows of $M_{i}$. We assume that, the number of rows in $M_{i}$ is the same for all nodes and equal to the number of orthogonal channels $(|C|)$.

Initially $M_{i}$ is unknown to player $i$, but this game can be played repeatedly in a sequence of game rounds $(1, \cdots T)$. To avoid channel oscillation in each round $t(t \in 1, \cdots T)$, the player plays a mixed strategy based on the weights $\left(w_{i, t}(c)\right)$ assigned to the rows of $M_{i}$. The probability of selecting the strategy $c$, is calculated as:

$$
P_{i, t}(c)=\frac{w_{i, t}(c)}{\sum_{c \in C} w_{i, t}(c)}
$$

At the beginning all weights are set to 1 , thus, the probability of selecting any channel is identical. After selecting a channel, the node gathers information from its neighbors and updates the loss that is suffered (equation (5)). Then, the weights are updated as:

$$
w_{i, t}(c)=w_{i, t-1}(c) \beta^{M_{i}\left(c, S_{-i}\right)}
$$

where $\beta$ is the game parameter in the range of $(0,1)$. A big $\beta$ introduces minor changes to the weights and the learner, follows the environment more accurate but slowly. Therefore it is applicable to a scenario where the environment changes less frequently. On contrary, a small $\beta$ imposes big changes in the weights, and introduces bigger error to the decision but adequate to a scenario with frequent changes. In our simulations we found that $\beta=0.2$ leads to better results (see Section VIII). We use the same $\beta$ for all players. Note that the best solution reached by the learner is not necessarily the Nash Equilibrium. It was shown that, multiplicative weights updates learning algorithm cannot work for Nash Equilibria in general bimatrix games [16].

\section{Channel Assignment Implementation}

Alg. 1 summarizes the implementation of the channel assignment previously described. Recall that the main idea of SICA is using available information on each node, gathered from its neighbors, and selecting the best channel by playing a game with mixed strategies. As explained in section V-A, the game is played in rounds that we shall 
refer to as channel assignment periods, and represent its duration by $T_{C A}$. Each node $i$ runs Alg. 1 at every $T_{C A}$.

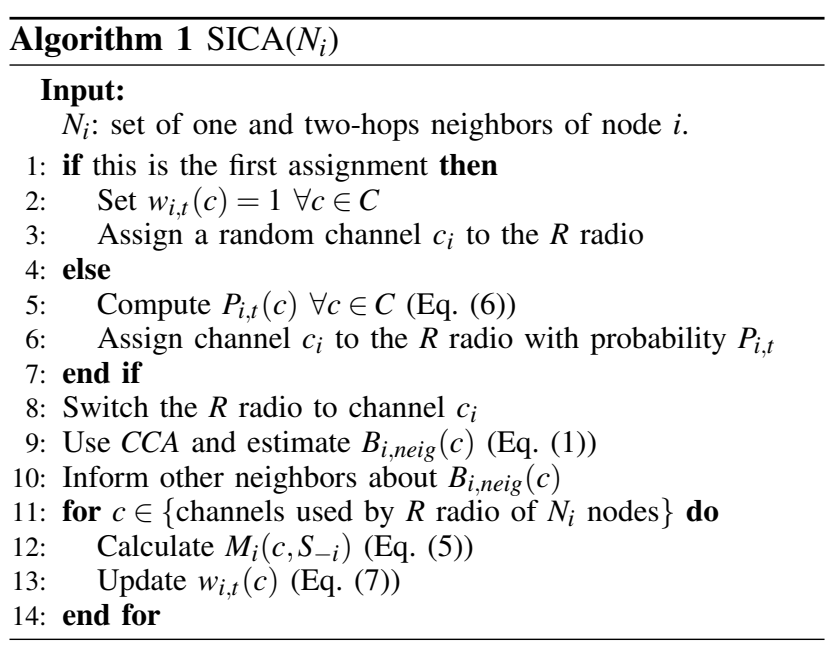

Four main reasons call for SICA to be an efficient channel assignment algorithm:

- Nodes are not required to have the perfect information about other players' strategies and loss functions.

- Nodes are supposed to be selfish players trying to occupy the best channels.

- It is not necessary for a node to estimate the external interference over all channels.In our proposal each node senses one channel and uses the information of its neighbors about other channels. Moreover the algorithm only estimates the interference over the channel of its $R$ radio.

- The proposed channel assignment eliminates the Channel Oscillation problem. This problem happens when some nodes find a channel empty and try to occupy it simultaneously, finally they will switch back when they find it busy by others. Playing a mixed strategy, as previously described, avoids channel oscillation since each node selects the destination channel randomly with a predefined probability.

\section{MESH NODES SYNCHRONIZATION MECHANISM}

Unlike Urban-X (see Section II) and many other CAs in the literature, in SICA there is no common channel between all nodes but the synchronization is achieved through exchanging messages. Since each node can assign a different channel to its receiving $(R)$ radio, the network topology may appear to be partitioned. To avoid network partitioning, nodes must be aware of the channels used by their neighbors' $R$ radios.A Node broadcasts Hello messages to report the channel of its $R$ radio to its neighbors.

It is not necessary to send Hello messages over all available channels, except when a new node joins the network or when a node stops receiving Hello messages from any neighbors. Once a node knows the channels used by the $R$ radios of its one-hop neighbors, then the node switches the $T$ radio to those channels and sends Hello messages every specific period of time.
After gathering information from the neighbors, a node may start transmitting data. One node may have packets to deliver to different neighbors on different channels. In our model each channel is associated with a queue. Packets are added to the corresponding queue according to the receiving channel of the neighbors. When the $T$ radio switches to each channel, it sends all or some of the packets in the associated queue. We use a different queue for Hello messages, which has higher priority than data packets' queue.

Due to space limitations we cannot give a more detailed description of the data delivery and channel switching mechanism, but they can be found in [17].

\section{PERFormance EVAluation}

In this section, we study the performance of the proposed channel assignment algorithm using ns-3 simulator [18] for 802.11-based multi-radio mesh networks. We use a network where the mesh routers initialize their routing tables using Shortest Path First (SPF), minimizing the number of hops. We assume a two ray ground propagation model with a radio range of $250 \mathrm{~m}$. Wireless nodes can tune their radio to any channel among 8 non-overlapping channels (according to IEEE 802.11a standard). CTS/RTS mechanism is disabled.

We compared SICA with another interference-aware channel assignment proposed in the literature called Urban-X [4]. Urban-X uses three radios for each node: an $R$ and $T$ radios, as in SICA, and a third radio which is tuned to a common channel for all nodes. The common channel stays unchanged through the life time of the network. Channel assignment in Urban-X takes into account the amount of flows a node has to send, and the estimated external interference over the channels. Nodes need to have information about the number of flows their neighbors have. Then Urban-X assigns a priority to each node based on the number of active flows it has, and nodes having higher priority have more chances to occupy the best channels (those with less traffic from external networks). Nodes broadcast control messages over the common channel up to two-hops neighbors. After switching to a channel, the $T$ radio remains there for a predefined period of time (40 ms). We've chosen Urban-X because it is a recent proposed distributed and interference aware channel assignment.

We've used a dual-radio network to evaluate SICA, while for Urban-X, we have added an extra radio for each node for the common channel. We have evaluated the performance of the protocols for different number of nodes which are placed in a grid topology. The traffic is generated by $100 \mathrm{kbps}$ CBR flows sent over vertical and horizontal directions in the grid.

The channels having interference from external networks are chosen randomly. We have done simulations using different number of channels. A channel with external interference is modeled as an on-off process, such that the channel is sensed busy and idle during the on and off states, respectively. The duration of the busy state has 


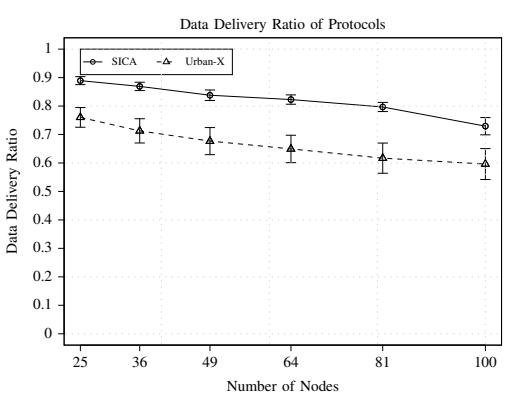

Figure 1: Data delivery ratio vs different number of nodes

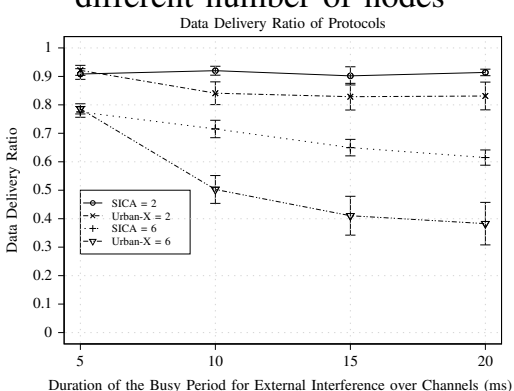

Figure 4: Data delivery ratio vs the number of channels that suffer external interference

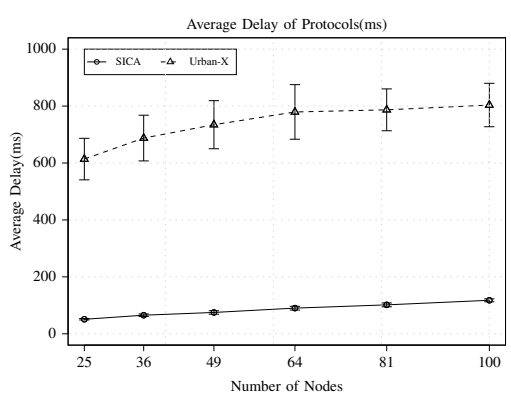

Figure 2: Average end to end delay vs different number of nodes

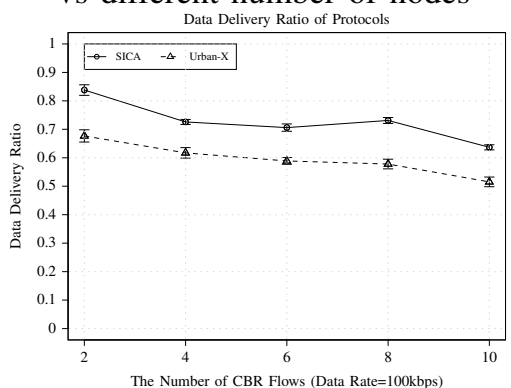

Figure 5: Data delivery ratio vs different CBR traffic loads

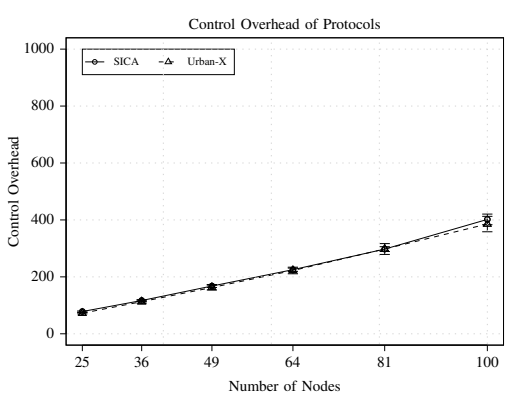

Figure 3: Control overhead vs different number of nodes

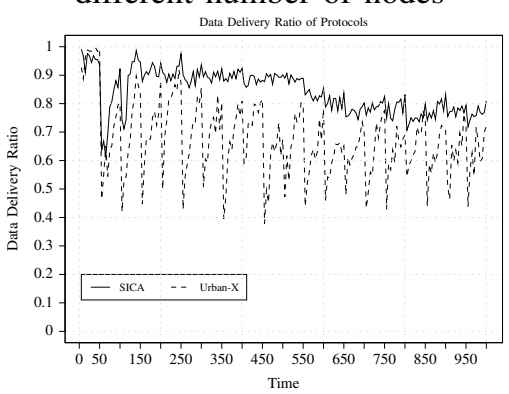

Figure 6: Data delivery ratio over time been fixed to a constant value, while the duration of the idle state is chosen exponentially distributed. The duration of the busy and idle periods have been varied to produce different interference loads. The detailed description of SICA parameters and the assigned values can be found in [17]. The Urban-X specific parameters are set according to the values given in [4].

\section{A. 802.11 Based Multi-Radio Performance}

We consider three network performance measures:

- Data delivery ratio: ratio of the total amount of data which is correctly received by the destinations, to the total amount of data packets transmitted by the sources.

- Average end to end delay: mean delay of the packets to reach the destination.

- Control overhead: ratio of the total number of control messages sent between nodes, to the total number of correctly received packets.

Fig. 1-3 shows the network performance for different number of nodes and two CBR traffic flows of $100 \mathrm{kbps}$. Every $50 \mathrm{~s}$, external interference is introduced over 4 channels chosen randomly. In these simulations the duration of busy state of the external interference is fixed to $10 \mathrm{~ms}$, while the mean duration of the idle state is $8 \mathrm{~ms}$. The results have been obtained averaging over 10 runs of $1000 \mathrm{~s}$ simulation time with different seeds. The error bars in the figures show $95 \%$ confidence intervals.

In Fig. 1 we can see that the delivery ratio is $10 \%$ higher in SICA than in Urban-X. This is a significant improvement, since Urban-X uses 3 radios and SICA uses only 2 . This result shows that the game theory approach used in the channel assignment of SICA outperforms the priority scheme used in Urban-X.

In Fig. 2 we can see that the average end to end delay is much lower in SICA than Urban-X. SICA leads to a lower delay because of the fast switching of the $T$ radio over all channels, while Urban-X keeps the $T$ radio in each channel for a predefined period of time, regardless of having data to send.

Fig. 3 shows that both protocols have a similar control overhead in terms of Hello messages. Urban-X uses a specific common radio for exchanging control messages, and each control message is sent over two hops. SICA, on the other hand, sends control messages only over those channels where a node has neighbors.

Fig. 4 compares the delivery probability obtained with SICA and Urban-X, varying the number of channels with external interference, and the load of the interference. The $\mathrm{x}$-axis of these figures shows the load of the external interference, which has been varied by changing the duration of the busy state of the interference between $5 \mathrm{~ms}$ and $20 \mathrm{~ms}$, and maintaining the mean duration of the idle state equal to $8 \mathrm{~ms}$. We used a $7 \times 7$ grid network and introduced interference over 2 and 6 channels (in the figure are distinguished using different line types). Fig. 4 shows that, even with a high interference load, the delivery ratio in SICA changes from 90 to $85 \%$, when the interference is increased from 2 to 6 channels. On the other hand, delivery ratio in Urban-X drops from 60 to $40 \%$. Thus, we conclude that, SICA is much more robust and less sensitive to the external interference than Urban-X.

Fig. 5 compares the delivery ratio obtained with SICA and Urban-X varying the number of CBR sources. We use 
a $7 \times 7$ grid network. The other network parameters are the same as in Fig. 1. Fig. 5, shows that SICA outperforms Urban-X, confirming the conclusions drawn from Fig. 1.

In order to have a more detailed view of the protocol's behavior, Fig. 6 shows the time evolution of the delivery ratio obtained with SICA and Urban-X. In this figure we have used a $7 \times 7$ grid network. The other parameters are the same as in Fig. 1. The values shown in the figure have been obtained repeating the simulation for 20 different random seeds and averaging the delivery ratio over $5 \mathrm{~s}$ periods. The figure shows that in SICA the delivery ratio is kept more stable than in Urban-X. Recall that every $50 \mathrm{~s}$ external interference is introduced over 4 channels chosen randomly. Fig. 6 shows that Urban-X has a considerable drop in delivery ratio at these time instants. SICA, on the contrary, is less sensitive to the changes of external interference, demonstrating that SICA adapts faster than Urban-X to external interference.

We have also investigated the sensitivity of SICA to $\gamma$ and $\beta$ tuning parameters of the game model (see section V). We omit the details here due to space constrains, but they can be found in [17]. The results show that the performance of SICA is not very sensitive to $\gamma$, and the best results are obtained with $\beta \approx 0.2$.

\section{Conclusions}

In this paper we have considered the channel assignment problem in multi-radio wireless mesh networks. We have proposed a new semi-dynamic protocol called SICA. SICA uses the game theory and online prediction concepts for a distributed channel selection where nodes do not have perfect knowledge about others strategies. We have done a performance evaluation comparing SICA with Urban$\mathrm{X}$, which is an adaptive channel assignment algorithm proposed in the literature. Simulation results show the efficiency of SICA in exploiting channel diversity for avoiding external interference and reducing the internal interference with only 2 radios per node. Moreover, even if Urban-X uses 3 radios per node, SICA outperforms Urban$\mathrm{X}$ in terms of delivery ratio and delay.

\section{ACKNOWLEDGMENTS}

This work was supported by the Spanish government and Generalitat de Catalunya through projects TIN201021378-C02-01 and 2009-SGR-1167, respectively, and by the European Commission through the NoE EuroNF. We also acknowledge Enric Monte Moreno for helpful discussions during the project.

\section{REFERENCES}

[1] M. K. Marina, S. R. Das, and A. P. Subramanian, "A topology control approach for utilizing multiple channels in multi-radio wireless mesh networks," Computer Networks, vol. 54, no. 2, pp. $241-256,2010$.

[2] M. Amiri Nezhad and L. Cerdà-Alabern, "Utility based channel assignment mechanism for multi radio mesh networks," in 8th ACM international workshop on Mobility management and wireless access, ser. MobiWac'10. New York, NY, USA: ACM, 2010, pp. 68-74.
[3] K. N. Ramachandran et al., "Interference-aware channel assignment in multi-radio wireless mesh networks," in INFOCOM, 2006, pp. 1-12.

[4] W. Kim et al., "Urban-x: Towards distributed channel assignment in cognitive multi-radio mesh networks," in IFIP Wireless Days, Venice, Italy, 2010.

[5] M. Felegyhazi, M. Cagalj, S. Bidokhti, and J.-P. Hubaux, "Non-cooperative multi-radio channel allocation in wireless networks," in INFOCOM, May 2007, pp. $1442-1450$.

[6] J. Crichigno, M. you Wu, and W. Shu, "Protocols and architectures for channel assignment in wireless mesh networks," Ad Hoc Networks, vol. 6, pp. 1051-1077, 2008.

[7] W. Si, S. Selvakennedy, and A. Y. Zomaya, "An overview of channel assignment methods for multi-radio multi-channel wireless mesh networks," J. Parallel Distrib. Comput., vol. 70, pp. 505-524, May 2010.

[8] L. Gao, X. Wang, Y. Xu, and W. Chen, "Distributed multiradio channel allocation in multi-hop ad hoc networks." in ICC'08, 2008, pp. 3156-3160.

[9] I. Shah, S. Jan, and K.-K. Loo, "Selfish flow games in non-cooperative multi-radio multi-channel wireless mesh networks with imperfect information," in ICWMC, 2010, pp. $219-225$.

[10] H.-K. Kim, C.-Y. Oh, and T.-J. Lee, "Fair and CollisionAware Multi-Channel Assignment Based on Game Theory for Wireless Multi-Hop Networks," IEICE Transactions on Communications, vol. 92, pp. 1282-1290, 2009.

[11] L. Kleinrock and F. Tobagi, "Packet switching in radio channels: Part i-carrier sense multiple-access modes and their throughput-delay characteristics," IEEE Trans. Commun., vol. 23, no. 12, pp. 1400-1416, 1975.

[12] P. Gupta and P. Kumar, "The capacity of wireless networks," IEEE Transactions on Information Theory, vol. 46 , no. 2, pp. $388-404$, Mar 2000.

[13] D. Murray, M. Dixon, and T. Koziniec, "Scanning delays in 802.11 networks," in NGMAST, 9 2007, pp. $255-260$.

[14] Y. Freund and R. E. Schapire, "Game theory, on-line prediction and boosting," in COLT. New York, NY, USA: ACM, 1996, pp. 325-332.

[15] — "Adaptive game playing using multiplicative weights," Games and Economic Behavior, vol. 29, no. 1-2, pp. 79-103, October 1999.

[16] C. Daskalakis et al., "On learning algorithms for nash equilibria," in Algorithmic Game Theory, 2010, pp. 114125 .

[17] M. Amiri-Nezhad and L. Cerdà-Alabern, "A SemiDynamic and Interference Aware Channel Assignment for Multi-Radio Multi-Channel Wireless Mesh Networks," Universitat Politecnica de Catalunya, Tech. Rep. UPCDAC-RR-CNDS-2011-3, 2011. [Online]. Available: http: //gsi.ac.upc.edu/apps/reports/2011/31/semi-ca.pdf

[18] ns-3 development team, "ns-3 manual (release ns-3.9)," 2011. 